\title{
氮/硫共掺杂对纤水镁石模板碳纳米管电化学性能的影响
}

\author{
刘芳芳 ${ }^{1}$ ，传秀云 ${ }^{1}$ ，杨 扬 ${ }^{1}$ ，李爱军 ${ }^{1,2}$
}

(1. 北京大学 地球与空间科学学院, 造山带与地壳演化教育部重点实验室, 北京 100871 ; 2. 北京金羽新能科技有 限公司，北京 100095)

摘 要: 以天然矿物纤水镁石为模板、蔗糖为碳源制备多孔碳纳米管，并以硫脲为氮、硫源，采用水热法制备氮/硫 共掺杂的碳纳米管。结果表明, 掺杂碳纳米管继承了纤水镁石模板的柱状结构, 呈现中空管状, 增大了模板炭的比 表面积和孔容。在 $6 \mathrm{~mol} \cdot \mathrm{L}^{-1} \mathrm{KOH}$ 电解液中, 电流密度为 $1 \mathrm{~A} \cdot \mathrm{g}^{-1}$ 时, 未掺杂碳纳米管的比电容为 $62.2 \mathrm{~F} \cdot \mathrm{g}^{-1}$, 氮掺 杂之后碳纳米管的比电容为 $97.0 \mathrm{~F} \cdot \mathrm{g}^{-1}$, 氮/硫共掺杂的碳纳米管比电容为 $172.0 \mathrm{~F} \cdot \mathrm{g}^{-1}$, 氮/硫共掺杂后碳纳米管的电 化学性能比未掺杂的提高近 3 倍; 循环 1000 次电容保持率达 $89 \%$, 说明掺/硫共掺杂碳纳米管具有良好的电化学性 能。此外, 组装的对称型超级电容器同样展示了良好的电容性能。

关 键 词: 超级电容器; 纤水镁石; 氮/硫共掺杂; 碳纳米管; 电化学性能 中图分类号: TQ127 文献标志码: A

\section{Influence of N/S Co-doping on Electrochemical Property of Brucite Template Carbon Nanotubes}

\author{
LIU Fangfang ${ }^{1}$, CHUAN Xiuyun ${ }^{1}$, YANG Yang ${ }^{1}$, LI Aijun ${ }^{1,2}$
}

(1. The Key Laboratory of Orogenic Belts and Crustal Evolution (MOE), School of Earth and Space Sciences, Peking University, Beijing 100871, China; 2. Golden Feature Science and Technology Ltd, Beijing 100095, China)

\begin{abstract}
N/S doped carbon nanotubes were prepared with natural mineral fibrous brucite as template, sucrose as carbon source, and thiourea as nitrogen and sulfur source. Experimental results indicate that the doped carbon nanotubes inherit the one-dimensional columnar structure of the fibrous brucite template. In addition, it presents a hollow tubular structure, which increases the specific surface area and pore volume of the template carbon. In $6 \mathrm{~mol} \cdot \mathrm{L}^{-1} \mathrm{KOH}$ electrolyte, the electrochemical performance significantly improves after doping. CNT-N/S presents a high specific capacity of $172.0 \mathrm{~F} \cdot \mathrm{g}^{-1}$ at current density of $1 \mathrm{~A} \cdot \mathrm{g}^{-1}$, higher than those of CNT $\left(62.2 \mathrm{~F} \cdot \mathrm{g}^{-1}\right)$ and

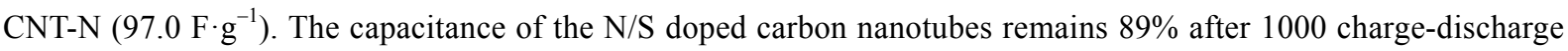
cycles. Furthermore, the assembled symmetrical supercapacitor also shows good capacitance performance.
\end{abstract}

Key words: supercapacitor; brucite; N/S co-doping; carbon nanotube; electrochemical performance

化石能源的枯竭与环境污染严重制约了社会经 济的发展 ${ }^{[1-3]}$ 。为了解决这一问题，开发新能源如风

收稿日期：2020-01-15; 收到修改稿日期：2020-12-03; 网络出版日期：2020-12-10

基金项目：国家自然科学基金重点项目(51774016，52074015); 北京大学开放测试基金(0000012321); 北京大学翁洪武科研 原创基金(WHW201803)

National Natural Science Foundation of China (51774016, 52074015); Test Fund of Peking University (0000012321); Weng Hongwu Original Research Fund of Peking University (WHW201803)

作者简介: 刘芳芳(1995-), 女, 博士研究生. E-mail: liuff4069@163.com

LIU Fangfang(1995-), female, PhD candidate. E-mail: liuff4069@163.com

通信作者：传秀云，教授. E-mail: xychuan@pku.edu.cn

CHUAN Xiuyun, professor. E-mail: xychuan@pku.edu.cn 
能、太阳能和氢能 ${ }^{[4]}$ 等, 以及高性能储能器件如超级 电容器、钠离子电池和钾离子电池等 ${ }^{[5]}$ 引起了广泛 关注 ${ }^{[6-8]}$ 。在各种储能器件中, 超级电容器因其循环 寿命长、功率密度高而被认为是最有应用前景的储 能装置之一 ${ }^{[9-12]}$ 。

根据储能机理, 超级电容器主要分为三种: (a) 在充放电过程中不发生化学反应, 靠电极表面电荷 的积累存储能量的双电层电容器; (b) 充放电过程中 在电极表面发生快速可逆氧化还原反应产生赝电容 的法拉第准电容器; (c) 兼具赝电容和双电层电容行 为的混合型超级电容器 ${ }^{[13-17]}$ 。

碳纳米管具有比表面积大、环境友好、物化性能 优异等优点, 是常见的双电层电容器的电极材料 ${ }^{[18]}$, 但是纯炭材料表面疏水、内阻较大等因素, 导致其 对实际容量的贡献较小 ${ }^{[19]}$ 。 B、N、P、S ${ }^{[20]}$ 等杂原 子掺杂是一种提高碳纳米管电化学性能简单有效的 方法。通过掺杂可以活化杂原子附近的 C 原子、显 著改善碳纳米管表面的润湿性, 此外, 引入含杂原 子官能团可以贡献噟电容, 从而提高炭材料的比电 容 $^{[21-25]}$, 其中氮化物 ${ }^{[26]}$ 、氮掺杂材料在析氢、储能 领域具有良好的应用前景。

纤水镁石是一种天然纤维状矿物, 具有丰富孔 道结构。我国陕西有世界上储量最大的纤水镁石矿 床 ${ }^{[27]}$, 成本低廉, 目前纤水镁石主要应用于阻燃耐 火材料、水泥基增强增韧填料、制取高纯氧化镁以 及抄纸等领域 ${ }^{[28]}$, 而在储能器件方面的应用较少。 本研究以天然矿物纤水镁石为模板、蔗糖为碳源, 制 备碳纳米管, 但是, 以纤水镁石制备的碳纳米管表 面疏水且比容量较低, 为了解决这一问题, 以硫脲为 氮/硫源, 采用简单易操作的水热法进一步制备了氮 /硫共掺杂碳纳米管, 以改善电化学性能。

\section{1 实验方法}

\section{1 模板法制备碳纳米管}

首先对纤水美石进行提纯分散 ${ }^{[29]}$, 将 $2 \mathrm{~g}$ 提纯 分散后的纤水镁石加入到 $200 \mathrm{~mL}$ 乙醇中, 剧烈摚 拌 $1 \mathrm{~h}$, 超声分散 $1 \mathrm{~h}$, 使纤水镁石均匀分散于乙醇 中, 然后加入 $4 \mathrm{~g}$ 蔗糖, 搅拌 $2 \mathrm{~h}$ 后, 抽真空。在 $80{ }^{\circ} \mathrm{C}$ 水浴条件下继续摚拌聚合 $8 \mathrm{~h}$, 待乙醇完全挥 发, 再将样品置于 $110{ }^{\circ} \mathrm{C}$ 烘箱中 $2 \mathrm{~h}$, 使混合物完全 干燥。将固体物质研磨成粉末, 置于坩埚中。接着 将其放入高温管式炉中, 在氩气氛围中 $450{ }^{\circ} \mathrm{C}$ 保温 $2.5 \mathrm{~h}$, 然后升温至 $750{ }^{\circ} \mathrm{C}$ 保温 $3 \mathrm{~h}$ (升温速率 $5{ }^{\circ} \mathrm{C} \cdot \mathrm{min}^{-1}$ )。降温后, 将所得样品移入烧杯中, 加入 质量分数为 $34 \%$ 的稀盐酸溶液(样品: 稀盐酸=
$1 \mathrm{~g}: 2.5 \mathrm{~mL}$ ), 充分搅拌 $24 \mathrm{~h}$, 去除模板, 抽滤水洗 至中性, 乙醇洗三次, 然后在 $110{ }^{\circ} \mathrm{C}$ 干燥 $12 \mathrm{~h}$, 得 到碳纳米管, 标记为 $\mathrm{CNT}$ 。

\section{2 氮掺杂碳纳米管的制备}

取 $1.5 \mathrm{~g}$ 尿素置于 $100 \mathrm{~mL}$ 烧杯中并加入 $50 \mathrm{~mL}$ 去离子水, 搅拌 $0.5 \mathrm{~h}$ 至尿素完全溶解, 加入 $0.05 \mathrm{~g}$ 模板炭, 搅拌 $0.5 \mathrm{~h}$ 至溶液混合均匀。然后将混合溶 液移入 $100 \mathrm{~mL}$ 聚四氟乙烯反应釜中, $200{ }^{\circ} \mathrm{C}$ 反应 $20 \mathrm{~h}$, 待冷却至室温后, 利用去离子水和乙醇洗涤 三次, $50{ }^{\circ} \mathrm{C}$ 干燥 $12 \mathrm{~h}$, 得到氮掺杂碳纳米管, 标记 为 CNT-N。

\section{3 氮/硫共掺杂碳纳米管的制备}

取 $1.0 \mathrm{~g}$ 硫脲置于 $100 \mathrm{~mL}$ 烧杯中, 加入 $50 \mathrm{~mL}$ 去 离子水, 摚拌 $0.5 \mathrm{~h}$ 后, 使硫艮完全溶解, 加入 $0.05 \mathrm{~g}$ 模板炭, 搅拌 $0.5 \mathrm{~h}$, 使悬浊液混合均匀, 然后将混 合溶液移入 $100 \mathrm{~mL}$ 聚四氟乙烯反应釜中, $200{ }^{\circ} \mathrm{C}$ 反 应 $20 \mathrm{~h}$, 待冷却至室温后, 利用去离子水和乙醇洗 涤三次, $50{ }^{\circ} \mathrm{C}$ 干燥 $12 \mathrm{~h}$, 得到氮/硫共掺杂碳纳米管, 标记为 CNT-N/S。制备流程见图 S1。

\section{4 电极的制备和超级电容器的组装}

在三电极体系中, 以金属铂片为对电极、录-氧 化永电极为参比电极, 在 $6 \mathrm{~mol} \cdot \mathrm{L}^{-1} \mathrm{KOH}$ 水溶液中 采用 $\mathrm{CHI} 660 \mathrm{E}$ 型电化学工作站对工作电极进行循 环伏安扫描 $(-0.8 \sim 0 \mathrm{~V})$ 、恒电流充放电 $(-0.8 \sim 0 \mathrm{~V})$ 、 电化学阻抗谱 $\left(0.1 \sim 10^{5} \mathrm{~Hz}\right)$ 测试。工作电极的制备: 将制备的样品、导电剂(乙炔黑)、聚四氟乙烯乳液 (PTFE, 60wt\%)按 8:1:1 的质量比混合, 加入适量 乙醇研磨得到粘稠状浆料, 将浆料均匀涂覆到泡沫 镍集流体上, 面积约 $1 \mathrm{~cm}^{2} 。 100{ }^{\circ} \mathrm{C}$ 真空干燥 $2 \mathrm{~h}$ 后, 使用油压机压制得到电极片(压力 $10 \mathrm{MPa}$ )。将压制 好的电极片放入 $6 \mathrm{~mol} \cdot \mathrm{L}^{-1} \mathrm{KOH}$ 溶液中, 在真空血. 中抽真空浸润 $0.5 \mathrm{~h}$, 将碳纳米管孔道中的气体排出, 得到最终的工作电极。根据恒流充放电曲线, 活 性物质的单电极质量比电容 $\left(C, \mathrm{~F} \cdot \mathrm{g}^{-1}\right)$ 按下面公式 计算:

$$
C=\frac{I \times \Delta t}{m \times \Delta U}
$$

式中, $I(\mathrm{~A})$ 为放电电流, $\Delta t(\mathrm{~s})$ 为放电时间, $m(\mathrm{~g})$ 为工 作电极活性物质质量, $\Delta U(\mathrm{~V})$ 为放电过程中的电位 窗口。

在两电极测试过程中, 以制备的样品为正负极, 采用 $6 \mathrm{~mol} \cdot \mathrm{L}^{-1} \mathrm{KOH}$ 作为电解液, 玻璃纤维为隔膜, 组装成 2032 型纽扣型对称电容器。恒流充放电、循 环伏安以及交流阻抗测试均在 CHI660E 型电化学 工作站上进行。 
正负极的制备方法与上面提到的三电极类似, 按质量比 $8: 1: 1$ 将制备的样品、乙炔黑及 PVDF 均匀分散于 NMP 中, 研磨得到粘稠状浆料, 将浆料 均匀涂覆到石墨纸上, $100{ }^{\circ} \mathrm{C}$ 真空干燥 $2 \mathrm{~h}$, 后切片 得到最终电极。根据恒流充放电曲线, 单电极的比 电容 $\left(C, \mathrm{~F} \cdot \mathrm{g}^{-1}\right)$ 按下面公式计算:

$$
C=\frac{4 \times I \times \Delta t}{m \times \Delta U}
$$

式中, $I 、 \Delta t$ 和 $\Delta U$ 代表的意义和(1)式中的相同, 其 中 $m(\mathrm{~g})$ 为正负极中活性物质的总质量。

实验原料和测试仪器见补充材料。

\section{2 结果和讨论}

\subsection{XRD 分析}

纤水镁石提纯分散前后的 X 射线衍射(XRD)图 谱见图 S2。提纯前后的样品均在 $2 \theta=20^{\circ}, 37^{\circ}, 50^{\circ}$ 和 $70^{\circ}$ 附近出现尖锐衍射峰, 与纤水镁石的标准衍 射峰相吻合(JCPDS\#07-0239), 分别对应其(001)、 (101)、(102)和(103)晶面。此外, 原矿中还出现了利 蛇纹石的特征衍射峰, 说明原矿中含有少量利蛇纹 石杂质, 这主要是因为纤水镁石原矿的围岩是蛇纹 岩, 在矿产露天开采时会引入围岩蛇纹石杂质。经 磁选酸洗提纯分散后，利蛇纹石的衍射峰基本消失， 得到了比较纯净的纤水镁石。

为了确定模板炭材料的物相和结晶程度, 对所 制备的三种模板炭碳纳米管 $(\mathrm{CNT})$ 、氮掺杂碳纳米 管 (CNT-N)和氮/硫共掺杂碳纳米管 $(\mathrm{CNT}-\mathrm{N} / \mathrm{S})$ 进行 XRD 测试(图 1)。三种多孔炭材料均表现出无定型 炭的特征, 在 $2 \theta=23^{\circ}$ 和 $44^{\circ}$ 左右出现两个宽衍射峰, 对应炭的(002)和(100)衍射峰。样品中纤水镁石的特 征衍射峰消失，说明模板被彻底去除。

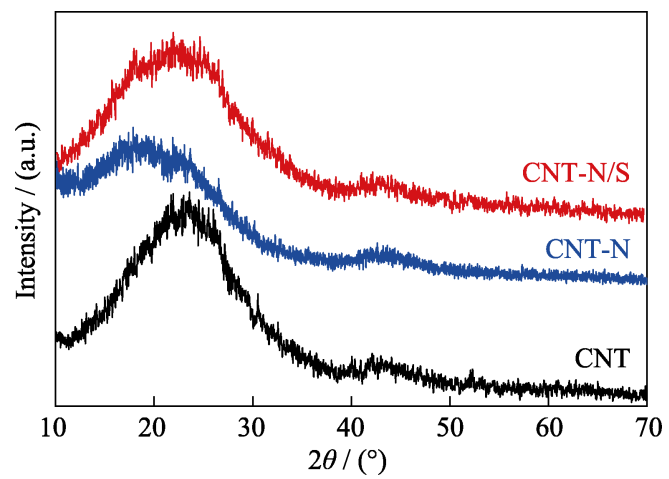

图 1 纤水镁石制备的模板纳米管 CNT、CNT-N、CNT-N/S 炭材料的 XRD 图谱

Fig. 1 XRD patterns of fibrous brucite templated carbon nanotube CNT, CNT-N and CNT-N/S

\section{2 拉曼光谱分析}

研究采用拉曼光谱表征以纤水镁石为模板制备 的 CNT、CNT-N 和 CNT-N/S 的结构有序程度。从 图 2 中可见, 三种模板炭材料在 $1590 \mathrm{~cm}^{-1}$ 附近均存 在尖的特征峰 ( $\mathrm{G}$ 峰), 该峰对应于 $E_{2 \mathrm{~g}}$ 对称振动峰. 在 $1342 \mathrm{~cm}^{-1}$ 附近的特征峰为 D 峰, 该峰是炭材料 的晶格缺陷引起的 ${ }^{[31]}$ 。其中 $\mathrm{D}$ 峰与 $\mathrm{G}$ 峰的强度比 $R\left(I_{\mathrm{D}} / I_{\mathrm{G}}\right)$ 可用来表征材料的石墨化程度, $R$ 越小, 表明 样品的石墨化程度越高 ${ }^{[32]}$ 。通过计算 CNT、CNT-N 和 CNT-N/S 的 $R$ 值分别为 $0.695 、 0.759$ 和 0.762 , 掺 杂后的炭材料的 $R$ 值显著增大, 表明引入杂原子使 炭材料无序度增加。

\subsection{SEM 和 TEM 形貌分析}

图 3 是纤水镁石模板及三种炭材料的扫描电镜 (SEM)和透射电镜 (TEM) 照片, 图 3(a,d)中纤水镁石 模板呈一维柱状结构。以其为模板制备的样品 CNT 很好地继承了模板的一维管状纳米结构(图 3(b,f))。 经过氮掺杂(图 3(c, g)) 和氮硫共掺杂(图 3(d, h)) 之后, 碳纳米管仍呈现一维管状纳米结构, 直径约 $50 \mathrm{~nm}$, 长度 1 2 $\mu \mathrm{m}$ 。 TEM 照片可以进一步验证三种材料 的一维管状结构, 此外三种样品均呈现中空管状结 构, 炭管的外径约为 $50 \mathrm{~nm}$, 内径约为 $44 \mathrm{~nm}$ 。碳纳 米管的介孔孔道结构为材料提供了较大的比表面积, 有利于电解液离子的快速传输。

\section{4 孔结构分析}

对 CNT, CNT-N 和 CNT-N/S 进行氮气吸脱附测 试, 表征孔结构特征。由图 4(a)可知, CNT, CNT-N 和 CNT-N/S 均属于IV 型氮气吸脱附曲线 ${ }^{[33]}$, 说明三 种炭材料含有较多中孔和大孔，在低压阶段仍有少 量吸附体积，表明材料中同时还含有少量微孔。因 此, CNT、CNT-N 和 CNT-N/S 均兼具微孔、中孔和 大孔多级孔道结构。对比图 4(a)可以发现, 在相对压

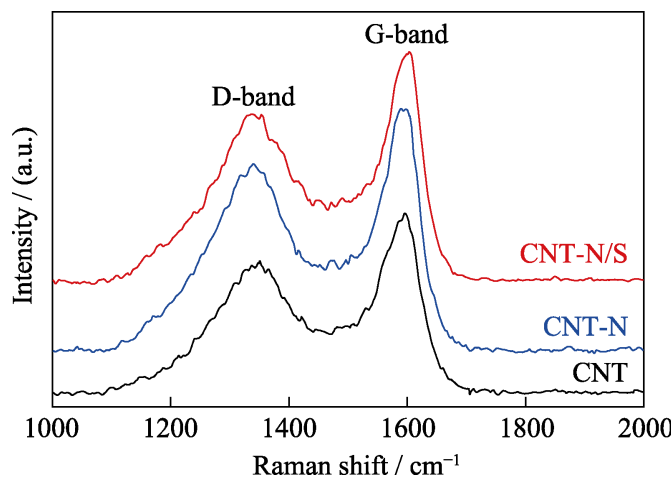

图 2 纤水镁石制备的模板纳米管 CNT、CNT-N 和 CNT-N/S 的拉曼光谱图

Fig. 2 Raman spectra of the fibrous brucite templated carbon nanotube CNT, CNT-N and CNT-N/S 
力较高时, 三者均存在回滞环, 主要归因于介孔的 毛细管凝聚作用。在相对压力较低 $\left(p / p_{0}<0.2\right)$ 时, 未 掺杂碳纳米管的吸附量始终大于掺杂后碳纳米管, 说明掺杂之后样品微孔含量略微下降, 与表 1 中孔 结构参数相一致, CNT-N/S 和 CNT-N 的比表面积和 孔体积为 $224.8 \mathrm{~m}^{2} \cdot \mathrm{g}^{-1} 、 0.267 \mathrm{~cm}^{3} \cdot \mathrm{g}^{-1}$ 和 $245.1 \mathrm{~m}^{2} \cdot \mathrm{g}^{-1}$ 、 $0.213 \mathrm{~cm}^{3} \cdot \mathrm{g}^{-1}$, 比未掺杂的 CNT $\left(505.5 \mathrm{~m}^{2} \cdot \mathrm{g}^{-1}\right.$ 和
$0.381 \mathrm{~cm}^{3} \cdot \mathrm{g}^{-1}$ )显著降低, 这主要是由于引入氮硫会 使部分孔隙堵塞。图 4(b) 是利用 Barrett-JoynerHalenda(BJH)模型得到的 CNT, CNT-N 和 CNT-N/S 的孔径-孔体积分布图, 可以看出, CNT, CNT-N 和 CNT-N/S 的孔径分布均较宽, 最大的吸附孔体积均 在 $45 \mathrm{~nm}$ 左右, 这主要是由于纤水镁石模板炭管的 内径约为 $44 \mathrm{~nm}$, 与 $\mathrm{TEM}$ 照片的结果一致。
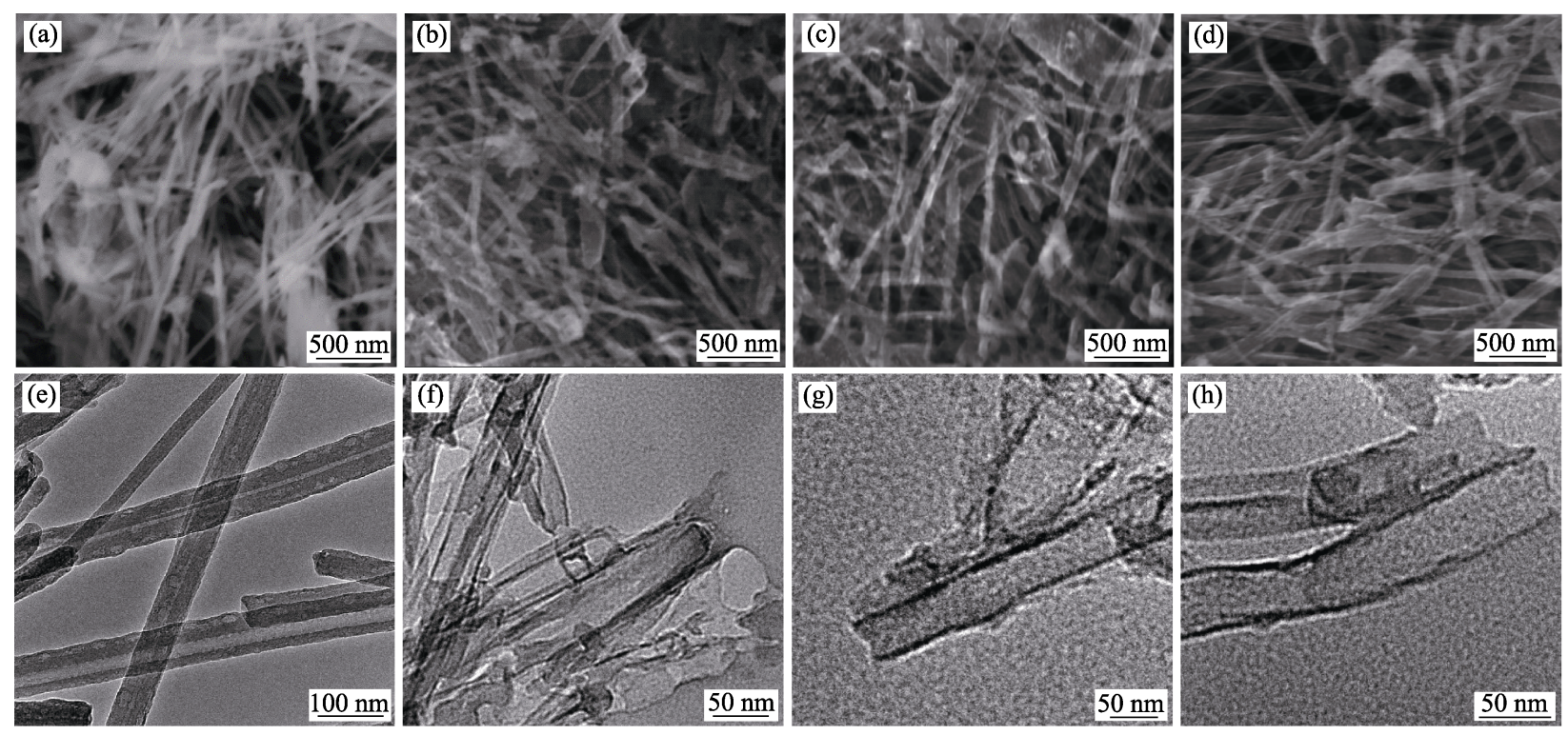

图 3 (a, e) 纤水镁石模板及纤水镁石制备的模板纳米管(b, f)CNT, (c, g)CNT-N 和 $(d, h) C N T-N / S$ 的(a d)SEM 和(e h)TEM 照片

Fig. 3 (a-d) SEM and (e-h) TEM images of (a, e) fibrous brucite, fibrous brucite templated carbon nanotube (b, f) CNT, (c, g) CNT-N, (d, h) CNT-N/S
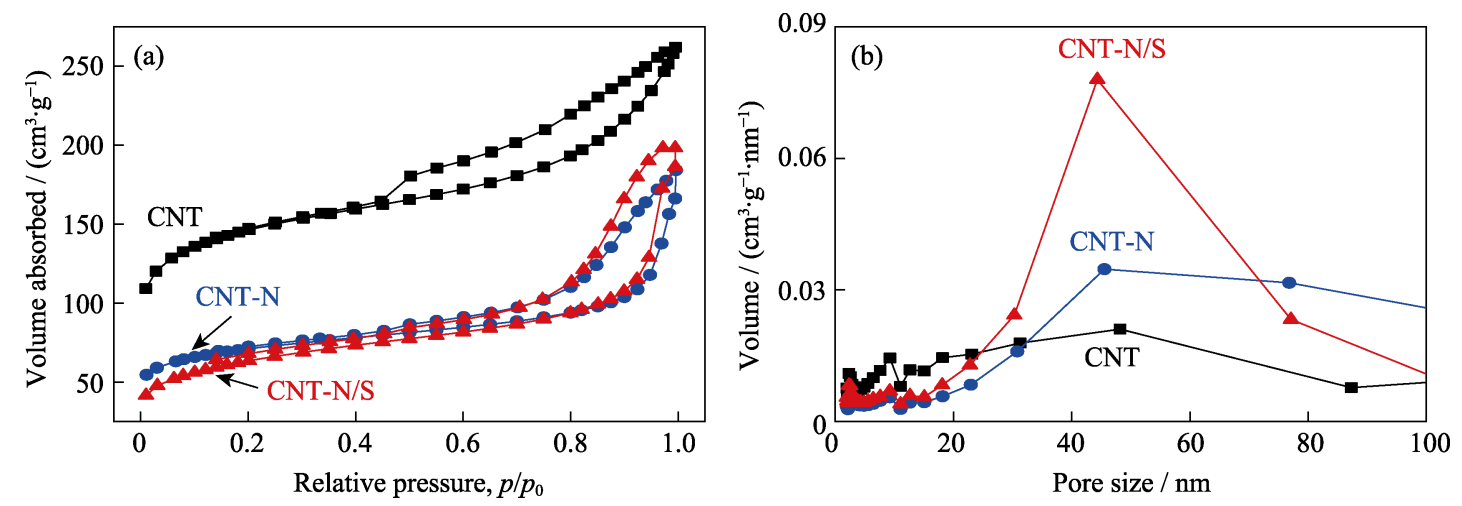

图 4 纤水镁石制备的模板纳米管 CNT, CNT-N 和 CNT-N/S 的(a)氮气吸脱附曲线和(b)BJH 孔径分布图

Fig. 4 (a) Nitrogen adsorption-desorption isotherms and corresponding (b) BJH pore size distribution curves of the Fibrous Brucite templated carbon nanotube CNT, CNT-N and CNT-N/S

表 1 纤水镁石制备的模板纳米管 CNT, CNT-N 和 CNT-N/S 的比表面积和孔结构参数

Table 1 Specific surface areas and pore structure parameters of fibrous brucite templated carbon nanotube CNT, CNT-N and CNT-N/S

\begin{tabular}{cccccc}
\hline Sample & $S_{\text {BET }} /\left(\mathrm{m}^{2} \cdot \mathrm{g}^{-1}\right)$ & $S_{\text {micropore }} /\left(\mathrm{m}^{2} \cdot \mathrm{g}^{-1}\right)$ & $V_{\text {total }} /\left(\mathrm{cm}^{3} \cdot \mathrm{g}^{-1}\right)$ & $V_{\text {mircropore }} /\left(\mathrm{cm}^{3} \cdot \mathrm{g}^{-1}\right)$ & $D_{\text {ap }} / \mathrm{nm}$ \\
\hline CNT & 505.5 & 273.5 & 0.381 & 0.125 & 3.02 \\
CNT-N & 245.1 & 131.3 & 0.213 & 0.060 & 3.48 \\
CNT-N/S & 224.8 & 63.8 & 0.267 & 0.028 & 4.75 \\
\hline
\end{tabular}




\section{$2.5 X$ 射线光电子能谱分析}

对 CNT、CNT-N 和 CNT-N/S 进行 X 射线光电 子能谱(XPS)测试, 分析其元素组成。由图 S3 可知, CNT-N/S 在 164.1、228.1、285.1、400.1 和 $533.1 \mathrm{eV}$ 出现特征峰, 分别对应于 $S 2 p 、 S 2 s 、 C 1 s 、 N 1 s$ 和 $\mathrm{O} 1 \mathrm{~s}$ 。内插图为 $\mathrm{N} 1 \mathrm{~s}$ 特征峰的局部放大图。根据积分 计算, 各个元素的含量见表 S1。图 5(a)是 CNT-N/S 的 $\mathrm{C} 1 \mathrm{~s}$ 的拟合谱图，可分成三个峰，分别位于 284.6 , 285.6 和 $287.3 \mathrm{eV}$, 对应 $\mathrm{sp}^{3}$ 杂化的碳 $(\mathrm{C}-\mathrm{C})$ 、与杂原 子成键的碳 $(\mathrm{C}-\mathrm{N} / \mathrm{C}-\mathrm{S})$ 和与氧原子成键的碳 $(\mathrm{C}=\mathrm{O})$ 。 图 5(b) 是 CNT-N/S 的 N1s 的拟合谱图, 位于 398.4, $400.5,401.0$ 和 $402.6 \mathrm{eV}$ 的四个峰, 分别对应吡啶型 氮(N-6), 吡咯型氮( $(\mathrm{N}-5)$, 类石墨型氮 $(\mathrm{N}-\mathrm{Q})$ 以及吡 啶氮氧化物 $(\mathrm{N}-\mathrm{X})$, 说明碳纳米管材料中成功掺杂 了氮元素 ${ }^{[34]}$ 。CNT-N/S 的 S2p 的拟合谱图(图 5(c)) 可以分成三个峰, 分别位于 $163.8,164.9$ 和 $168.7 \mathrm{eV}$, 其中 163.8 和 $164.9 \mathrm{eV}$ 两个峰对应 $\mathrm{C}-\mathrm{S}$ 键, $168.7 \mathrm{eV}$ 的峰对应于- $\mathrm{SO}_{n}$-基团, 表明成功掺杂了硫元素。掺 杂的 $\mathrm{N} 、 \mathrm{~S}$ 元素既可以活化附近的碳原子、增强导 电性, 还可以产生大量的亲水位点、改善材料表面 的润湿性、降低电解液离子在炭材料中的扩散阻力、 提高炭材料的表面利用率, 从而使比电容增加, 并 提供噟电容 ${ }^{[19]}$ 。

\section{6 电化学性能分析}

对样品进行三电极测试, 表征电极材料的电化
学性能。图 S4 (a c) 分别为 CNT、CNT-N 和 CNT-N/S 电极在不同扫描速率下的循环伏安 $(\mathrm{CV})$ 曲线。随着 扫速提高, 三个样品的 $\mathrm{CV}$ 曲线变形较小, 表现出 较理想的双电层电容特性以及良好的充放电可逆 性。CNT-N/S 电极的 CV 曲线中有一对弱氧化还原峰, 归因于引入含杂原子 N/S 官能团产生的赝电容 ${ }^{[19]}$ 。 这表明除了典型的双电层电容特性, CNT-N/S 电极 材料还出现了赝电容, 所以总电容显著提高。图 6(a) 对比了在 $100 \mathrm{mV} \cdot \mathrm{s}^{-1}$ 扫速下三者的循环伏安曲线, CNT-N/S、CNT-N 和 CNT 电极的循环伏安曲线围成 的面积依次减小, 说明质量比电容依次减小。图 S4 $(d \sim f)$ 分别是 CNT、CNT-N 和 CNT-N/S 电极在不同 电流密度下 $\left(0.5 \sim 10 \mathrm{~A} \cdot \mathrm{g}^{-1}\right)$ 的恒电流充放电 $(\mathrm{GCD})$ 曲 线。图 6(b)为 CNT, CNT-N 和 CNT-N/S 三个电极在 $1 \mathrm{~A} \cdot \mathrm{g}^{-1}$ 电流密度下的恒流充放电曲线, 可以看出, CNT 和 CNT-N 电极基本呈对称的三角形, 而 CNT$\mathrm{N} / \mathrm{S}$ 电极呈非线性特征, 偏离理想的对称三角形, 可能与其赝电容有关。根据公式(1)计算得到在电流 密度为 $1 \mathrm{~A} \cdot \mathrm{g}^{-1}$ 时, CNT、CNT-N 和 CNT-N/S 电极的 质量比电容分别为 $62.2,97.0$ 和 $172.0 \mathrm{~F} \cdot \mathrm{g}^{-1}$ 。在相同 的电流密度下, CNT-N/S 的质量比电容大于 CNT-N 和 $\mathrm{CNT}$, 这与 $\mathrm{CV}$ 曲线的结果一致。

图 6(c) 是 CNT, CNT-N 和 CNT-N/S 电极的循环 稳定性曲线, 在 $1 \mathrm{~A} \cdot \mathrm{g}^{-1}$ 的电流密度下经过 1000 次循 环充放电后，三种材料的比电容保持率分别为 $89.0 \%$,
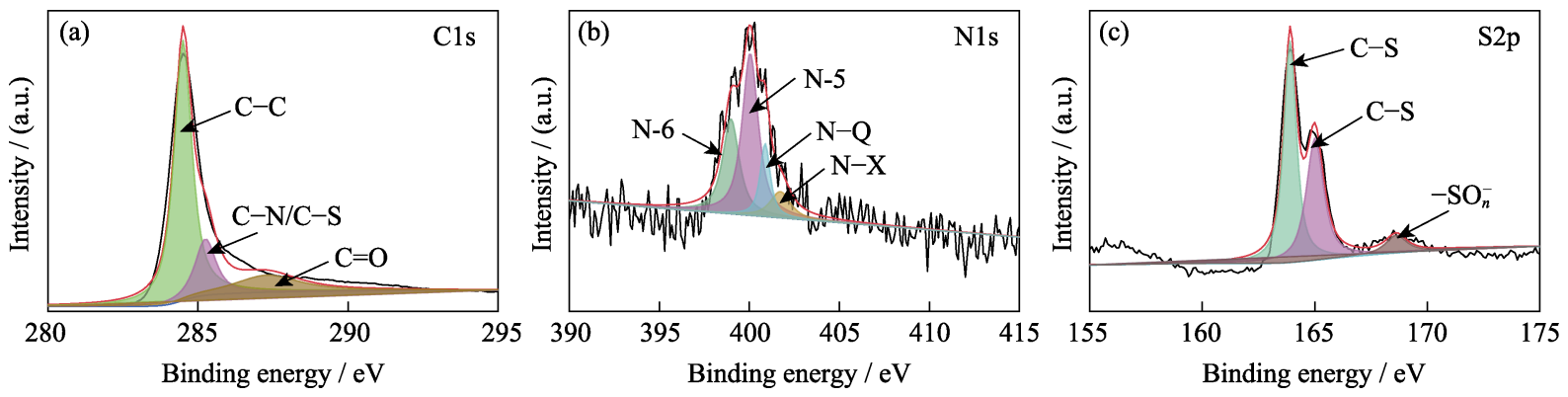

图 $5 \mathrm{CNT}-\mathrm{N} / \mathrm{S}$ 的(a)XPS C1s, (b)N1s 和(c)S2p 拟合谱

Fig. 5 Fitting curves of (a) C1s, (b) N1s and (c) S2p for CNT-N/S
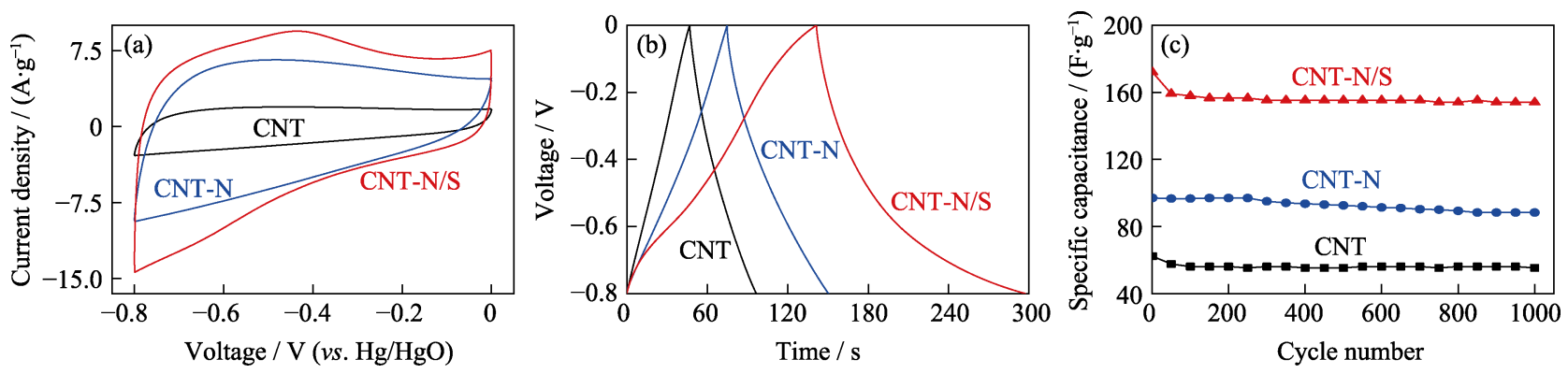

图 $6 \mathrm{CNT} 、 \mathrm{CNT}-\mathrm{N}$ 和 CNT-N/S 电极(a)在 $100 \mathrm{mV} \cdot \mathrm{s}^{-1}$ 下的循环伏安曲线,

(b)在 $1 \mathrm{~A} \cdot \mathrm{g}^{-1}$ 下的恒流充放电曲线, (c)在 $1 \mathrm{~A} \cdot \mathrm{g}^{-1}$ 下的循环稳定性曲线

Fig. 6 (a) CV curves at $100 \mathrm{mV} \cdot \mathrm{s}^{-1}$, (b) GCD curves at $1 \mathrm{~A} \cdot \mathrm{g}^{-1}$, (c) cycling performance at $1 \mathrm{~A} \cdot \mathrm{g}^{-1} \mathrm{of} C N T$, CNT-N and CNT-N/S 
91.0\%, 89.0\%, 均表现出优异的循环稳定性。图 S4(g) 给出了 CNT, CNT-N 和 CNT-N/S 电极的 Nyquist 曲 线。在高频区, Nyquist 曲线与 $x$ 轴的截距对应材料 材料内部电阻 ${ }^{[21]}$ 。CNT-N 和 CNT-N/S 电极的内部 电阻均小于 CNT 电极, 说明引入杂原子可以改善碳 纳米管表面的润湿性, 减小电解质离子在电极孔道 中的扩散阻力 ${ }^{[19]}$ 。在中频区, CNT-N 和 CNT-N/S 电 极的电荷转移电阻 $\left(R_{\mathrm{ct}}\right)^{[35]}$ 大于 $\mathrm{CNT}$ 电极, 这主要是 由于引入杂原子堵塞了碳纳米管材料的部分孔隙, 阻碍了电荷的运移, 导致其电荷转移电阻较高。此 外, 拟合计算的 CNT、CNT-N 和 CNT-N/S 的溶液电 阻 $\left(R_{\mathrm{s}}\right)$ 分别为 $2.89 、 1.67$ 和 $1.45 \Omega$ 。

为了进一步考察制备样品的电化学性能, 将样 品组装成对称型超级电容器(CNT//CNT、CNT-N// CNT-N 和 CNT-N/S//CNT-N/S), 其结构示意图见 图 S5(a)。图 S5(b d) 分别为 CNT//CNT、CNT-N//CNT-N 和 CNT-N/S//CNT-N/S 在不同扫描速率下的循环伏 安 $(\mathrm{CV})$ 曲线。随着扫速增加, 三者的 $\mathrm{CV}$ 曲线变形 较小, 均表现出较为理想的双电层电容特性以及良 好的充放电可逆性, 其中 CNT-N/S//CNT-N/S 的 CV 曲线最接近矩形, 说明其具有最理想的电容性能。

图 S5(e $\mathrm{g}$ ) 分别是 $\mathrm{CNT} / / \mathrm{CNT} 、 \mathrm{CNT}-\mathrm{N} / / \mathrm{CNT}-\mathrm{N}$ 和 CNT-N/S//CNT-N/S 在不同电流密度 $\left(0.5 \sim 10 \mathrm{~A} \cdot \mathrm{g}^{-1}\right)$ 下的恒流充放电(GCD) 曲线, 器件的恒流充放电曲 线有明显的非线性特征, 表明存在赝电容。根据公 式(2), 计算得到器件的比电容, 见图 7(a)。当电流密 度从 0.5 增加到 $10 \mathrm{~A} \cdot \mathrm{g}^{-1}$ (图 7(a)) 时, $\mathrm{CNT} / / \mathrm{CNT}$ 、 CNT-N//CNT-N 和 CNT-N/S//CNT-N/S 的电容保持率 依次为 $32 \% 、 42 \%$ 和 $64 \%$, 氮/硫共掺杂的样品具有 较好的倍率性能。对称型超级电容器在 $2 \mathrm{~A} \cdot \mathrm{g}^{-1}$ 下的 循环性能见图 7(b), 在 1000 次充放电后, 对称型超 级电容器的电容保持率均 $\geqslant 90 \%$, 表明其具有良好
的循环稳定性。采用电化学阻抗谱(EIS)测试探究对 称型超级电容器的电化学特性, 结果如图 7(c) 所示, 在低频区谱图均显示为直线, 表现出电容行为的特 征, 其中 CNT-N/S//CNT-N/S 的直线斜率最大, 说明 其扩散系数较大。Nyquist 曲线与 $x$ 轴的截距表示等 效串联电阻(ESR), 如图 7(c)的内插图所示, CNT// CNT、CNT-N//CNT-N 和 CNT-N/S//CNT-N/S 的 ESR 分别为 $0.58 、 1.58 、 0.66 \Omega$ 。

\section{3 结论}

以蔗糖为碳源、纤水美石为模板、尿素为氮源、 硫脲为氮/硫源, 采用水热法制备掺杂碳纳米管。掺 杂碳纳米管继承了纤水镁石模板的一维柱状结构, 并呈现多级孔道结构。掺杂后材料的无序度、比表 面积和孔容增加。对掺杂前后的碳纳米管进行电化 学性能研究, 结果表明: 在三电极体系中, 电流密 度为 $1 \mathrm{~A} \cdot \mathrm{g}^{-1}$ 时, 氮/硫共掺杂碳纳米管比容量达到 $172.0 \mathrm{~F} \cdot \mathrm{g}^{-1}$, 相比于未掺杂碳纳米管 $\left(62.2 \mathrm{~F} \cdot \mathrm{g}^{-1}\right)$ 和 氮掺杂碳纳米管 $\left(97.0 \mathrm{~F} \cdot \mathrm{g}^{-1}\right)$ 显著提高。循环 1000 次 后电容保持率达 $89 \%$, 说明氮/硫共掺杂碳纳米管具 有良好的循环稳定性。在两电极体系中, 以 $\mathrm{CNT}$ 、 CNT-N 和 CNT-N/S 分别组装成对称型超级电容器, 在电流密度为 $1 \mathrm{~A} \cdot \mathrm{g}^{-1}$ 时, CNT//CNT、CNT-N//CNT$\mathrm{N}$ 和 CNT-N/S//CNT-N/S 的比电容分别为 $42.7 、 76.8$ 和 $150.0 \mathrm{~F} \cdot \mathrm{g}^{-1}$, 说明掺杂后的模板炭材料具有良好 的电化学性能, 应用潜力巨大。

\section{补充材料}

本文相关补充材料可登陆 https://doi.org/10.15541/ jim20200033 查看。
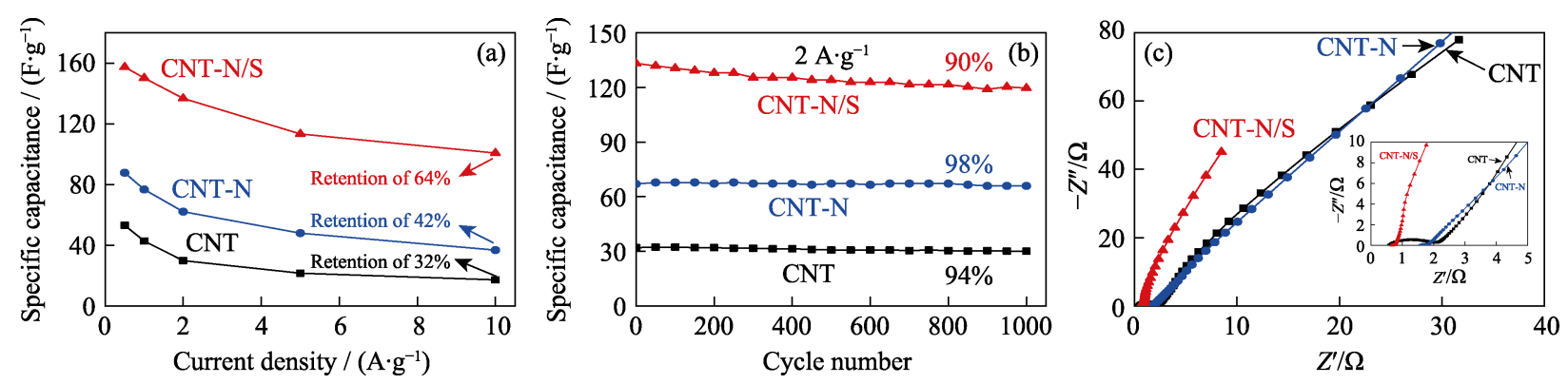

图 $7 \mathrm{CNT} / / \mathrm{CNT} 、 \mathrm{CNT}-\mathrm{N} / / \mathrm{CNT}-\mathrm{N}$ 和 CNT-N/S//CNT-N/S (a)在不同电流密度下的比电容曲线, (b)在 $2 \mathrm{~A} \cdot \mathrm{g}^{-1}$ 下的循环稳定性曲线和(c)Nyquist 曲线(插图为高频区域局部放大图)

Fig. 7 (a) Specific capacitance versus current density, (b) cycling performance at $2 \mathrm{~A} \cdot \mathrm{g}^{-1}$ and (c) Nyquist plots of CNT//CNT, CNT-N//CNT-N and CNT-N/S//CNT-N/S with insert in (c) showing the corresponding enlarged view of the high frequency 


\section{参考文献:}

[1] 向治华, 古庭梵, 黄友金. 能源转型发展的需求与创新模式分 析. 新型工业化, 2019, 9(7): 15-22.

[2] LI P, CHEN W. Recent advances in one-dimensional nanostructures for energy electrocatalysis. Chinese Journal of Catalysis, 2019, 40(1): 4-22.

[3] 梁启超, 乔芬, 杨健, 等. 太阳能电池的研究现状与进展. 中国 材料进展, 2019, 38(5): 505-511.

[4] LUO S W, GU R, SHI P H, et al. $\pi^{-} \pi$ interaction boosts catalytic oxygen evolution by self-supporting metal-organic frameworks. Journal of Power Sources, 2020, 448: 227406.

[5] DU J C, GAO S S, SHI P H, et al. Three-dimensional carbonaceous for potassium ion batteries anode to boost rate and cycle life performance. Journal of Power Sources, 2020, 451: 227727.

[6] JIAO Y, HONG W Z, LI P Y, et al. Metal-organic framework derived $\mathrm{Ni} / \mathrm{NiO}$ micro-particles with subtle lattice distortions for highperformance electrocatalyst and supercapacitor. Applied Catalysis B: Environmental, 2019, 224: 732-739.

[7] MA Y, HOU C P, ZHANG H P, et al. Three-dimensional core-shell $\mathrm{Fe}_{3} \mathrm{O}_{4} /$ polyaniline coaxial heterogeneous nanonets: preparation and high performance supercapacitor electrodes. Electrochimica acta, 2019, 315: 114-123.

[8] RAN F T, YANG X B, SHAO L, et al. Recent progress in carbonbased nanoarchitectures for advanced supercapacitors. Advanced Composites and Hybrid Materials, 2018, 1(1): 32-55.

[9] 黄兴兰. 超级电容器活性炭材料的研究进展. 东方电气评论, 2019, 33(2): 11-14+25.

[10] ZHANG S W, YIN B S, LIU X X, et al. A high energy density aqueous hybrid supercapacitor with widened potential window through multi approaches. Nano Energy, 2019, 59: 41-49.

[11] 季辰辰, 米红宇, 杨生春. 超级电容器在器件设计以及材料合 成的研究进展. 科学通报, 2019, 64(1): 9-34.

[12] 盛利成, 李芹, 董丽敏, 等. 基于碳材料的超级电容器电极材料 的研究现状. 电源技术, 2019, 43(7): 1241-1244.

[13] DA Y M, LIU J X, ZHOU L, et al. Engineering 2D architectures toward high-performance micro-supercapacitors. Advanced Materials, 2019, 31(1): 1802793 .

[14] 左宋林, 王永芳, 张秋红. 活性炭作为电能储存与能源转化材 料的研究进展. 林业工程学报, 2018, 3(4): 1-11.

[15] WASEEM RAZA, FAIZAN ALI, NADEEM RAZA, et al. Recent advancements in supercapacitor technology. Nano Energy, 2018, 52: $441-473$.

[16] 罗玉馨, 董小风, 罗梦琪, 等. 超级电容器电极材料及储能机理. 化工设计通讯, 2017, 43(5): 183+196.

[17] 李春花, 姚春梅, 吕启松, 等. 超级电容器储能机理. 科技经济 导刊, 2016(7): 107.

[18] ZHAI Y P, DOU Y Q, ZHAO D Y, et al. Carbon materials for chemical capacitive energy storage. Advanced Materials, 2011, 23(42): 4828-4850.
[19] 冯晨辰, 吴爱民, 黄吴. 超级电容器电极用 N-掺杂多孔碳材料 的研究进展. 材料导报, 2016, 30(1): 143-149.

[20] ABBAS QAISAR, RAZA RIZWAN, SHABBIR IMRAN, et al. Heteroatom doped high porosity carbon nanomaterials as electrodes for energy storage in electrochemical capacitors: a review. Journal of Science: Advanced Materials and Devices, 2019, 4(3): 341-352.

[21] 范景彪, 李志伟, 骆建敏, 等. 基于碳酸镁模板的氮掺杂碳纳米 管的制备及其电化学性能. 材料研究学报, 2018, 32(8): 599-606.

[22] 郭坤琨, 陈鹏, 李柱. 氮掺杂多孔碳的制备及其电化学性能研 究. 湖南大学学报(自然科学版), 2018, 45(6): 78-84.

[23] 蒋晓薇, 谭诗宇, 蔡冬琦, 等. 氮、磷共掺杂多孔类石墨烯碳的 制备及其电化学析氢性能. 海南师范大学学报(自然科学版), 2018, 31(2): 125-130.

[24] 王桂强，刘洁琼，董伟楠，等.氮/硫共掺杂多孔碳纳米片的制 备及其电化学性能. 物理学报, 2018, 67(23): 276-284.

[25] 钟彩丽, 陈梁, 李梦雪, 等. 介孔型氮硫双掺杂石墨烯的制备及 其电化学性能研究. 湖南理工学院学报(自然科学版), 2019 , 32(1): 43-47.

[26] GONG S Q, JIANG Z J, SHI P $\mathrm{H}$, et al. Noble-metal-free heterostructure for efficient hydrogen evolution in visible region: molybdenum nitride/ultrathin graphitic carbon nitride. Applied Catalysis B: Environmental, 2018, 238: 318-327.

[27] 崔义发. 陕南黑木林纤维水镁石矿地质特征及应用前景浅析. 化工矿产地质, 2011, 33(3): 155-160+188.

[28] 刘淑鹏, 袁继祖, 唐靖炎, 等. 纤维水镁石的研究进展与应用前 景. 矿业快报, 2007(4): 14-17.

[29] 曹曦, 传秀云. 纤水镁石的提纯与改性. 矿物学报, 2012, 32(S1): $85-86$.

[30] MIAO L, ZHU D Z, LIU M X, et al. Cooking carbon with protic salt: nitrogen and sulfur self-doped porous carbon nanosheets for supercapacitors. Chemical Engineering Journal, 2018, 347: 233-242.

[31] WANG Y H, LIU R N, TIAN Y D, et al. Heteroatoms-doped hierarchical porous carbon derived from chitin for flexible all-solidstate symmetric supercapacitors. Chemical Engineering Journal, 2020, 384: 123263

[32] 曹犧, 传秀云, 李爱军, 等. 纳米纤维矿物纤蛇纹石为模板合成 碳纳米管及其在超级电容器中的应用. 新型炭材料, 2018, 33(3): 229-236.

[33] SING KSW, EVERETT D H, HAUL RAW, et al. Reporting physisorption data for gas solid systems with special reference to the determination of surface-area and porosity. Pure and Applied Chemistry, 1985, 57(4): 603-619.

[34] MIAO L, ZHU D Z, LIU M X, et al. N, S co-doped hierarchical porous carbon rods derived from protic salt: facile synthesis for high energy density supercapacitors. Electrochimica Acta, 2018, 274: $378-388$

[35] WANG W H, WANG X D. Investigation of Ir-modified carbon felt as the positive electrode of an all-vanadium redox flow battery. Electrochimica Acta, 2007, 52(24): 6755-6762. 
补充材料:

\title{
氮/硫共掺杂对纤水镁石模板碳纳米管电化学性能的影响
}

\author{
刘芳芳 ${ }^{1}$, 传秀云 ${ }^{1}$, 杨 扬 $^{1}$, 李爱军 ${ }^{1,2}$
}

(1. 北京大学地球与空间科学学院, 造山带与地壳演化教育部重点实验室, 北京 100871 ; 2. 北京金羽新能科技有 限公司，北京 100095)

\section{实验方法}

\section{实验试剂}

纤水镁石 $\left(\mathrm{Mg}_{0.994} \mathrm{Fe}^{3+}{ }_{0.007} \mathrm{Mn}_{0.002} \mathrm{Ni}_{0.001}\right)_{1.001}$ $(\mathrm{OH})_{2.009}$, 采集于陕南宁强黑木林纤水镁石矿, 二 辛基磺基琥珀酸钠盐(国药集团化学试剂有限公司, $\geqslant 73.8 \%$ ), 尿素(西陇科学股份有限公司， $\geqslant 99 \%$ ), 硫脲(西陇科学股份有限公司, $\geqslant 99 \%$ ), 蔗糖(国药 集团化学试剂有限公司, $\geqslant 99 \%$ ), 盐酸(西陇科学股 份有限公司, $36 \%$ ), 氢氧化钾(西陇科学股份有限公 司, $\geqslant 85 \%$ ), 无水乙醇(现代东方(北京)科技发展有 限公司)和去离子水。

\section{测试仪器}

采用日本理学公司的 RigakuD/max 2400 X射线 衍射仪 $(\mathrm{CuK \alpha}, \lambda=0.15406 \mathrm{~nm}$, 靶电流 $100 \mathrm{~mA}$, 靶电 压 $40 \mathrm{kV}$, 步长 $0.02^{\circ}$, 扫描速度 $4\left(^{\circ}\right) \cdot \mathrm{min}^{-1}$, 扫描范 围为 $2 \theta=5^{\circ} \sim 70^{\circ}$ ) 进行 $\mathrm{X}$ 射线衍射分析。采用英国 Renishaw 公司的 RM-1000 型激光拉曼光谱仪(氦氞 激光器, 波长 $632.8 \mathrm{~nm}$, 分辨率 $1 \sim 2 \mathrm{~cm}^{-1}$ ) 进行激光 拉曼光谱分析。采用 FEI 公司的 NovaNanoSEM430 (加速电压 $15 \mathrm{kV}$, 束流 $176 \mu \mathrm{A}$ )进行场发射扫描电子 显微分析。采用美国 Micrometer 公司的 ASAP2010 氮气吸附-脱附测试仪进行氮气吸脱附测试, 测试 前在 $120{ }^{\circ} \mathrm{C}$ 脱气 $12 \mathrm{~h}$, 测试温度为 $-196{ }^{\circ} \mathrm{C}$ 。比表面 积用多点式 BET 法测定, 孔径分布利用非定域密度

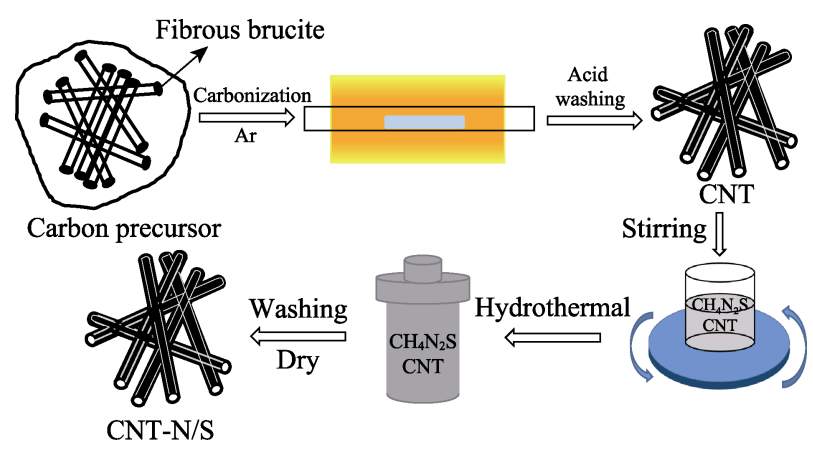

图 S1 氮硫共掺杂碳纳米管(CNT-N/S)的制备流程示意图

Fig. S1 Schematic of preparation process for CNT-N/S
泛函理论测定 ${ }^{[1]}$ 。采用岛津集团英国 Kratos 公司的 AXIS Supra X 射线光电子能谱仪(用单色的铝/银靶 $X$ 射线为光源, $40 \mathrm{kV}, 40 \mathrm{~mA}$ )进行 $X$ 射线光电子能 谱分析。

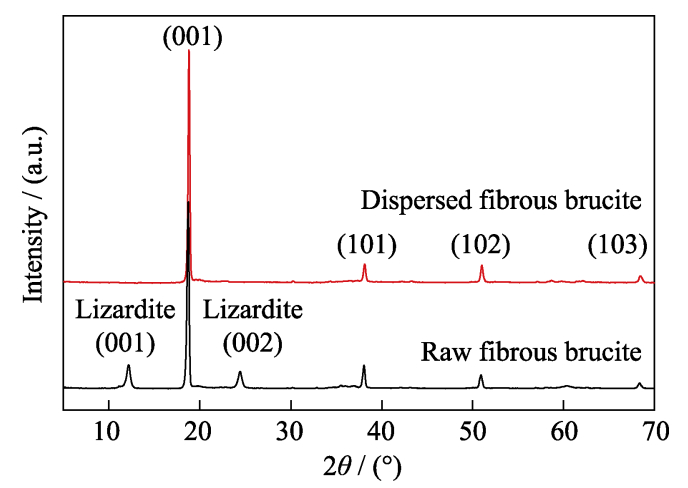

图 S2 纤水镁石原矿与提纯分散纤水镁石的 XRD 图谱

Fig. S2 XRD patterns of raw and dispersed Fibrous Brucite

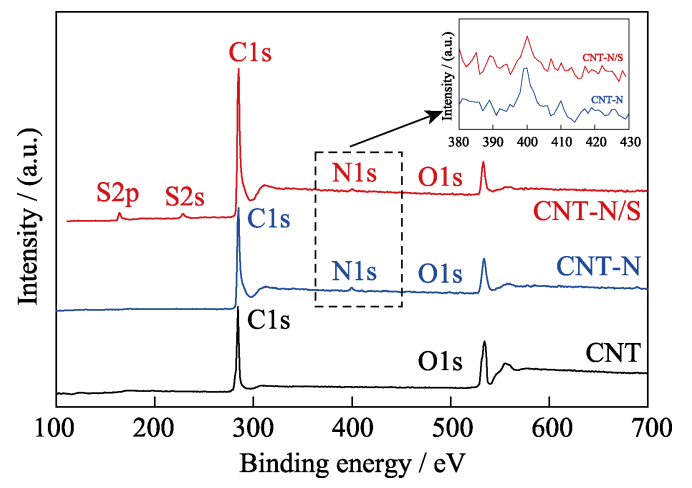

图 S3 纤水镁石制备的模板纳米管 CNT、CNT-N 和 CNT-N/S 的 XPS 全谱(内插图为 N1s 特征峰的局部放大图)

Fig. S3 XPS spectra with the enlarged view of N1s peaks in the insert of Fibrous Brucite templated carbon nanotube CNT, CNT-N and CNT-N/S

\section{表 S1 纤水镁石制备的模板纳米管 CNT、 CNT-N 和 CNT-N/S 的元素组成}

Table S1 Elemental composition of Fibrous Brucite templated carbon nanotube CNT, CNT-N and CNT-N/S

\begin{tabular}{ccccc}
\hline Sample & C/at $\%$ & O/at $\%$ & N/at $\%$ & S/at $\%$ \\
\hline CNT & 87.34 & 12.66 & - & - \\
CNT-N & 84.93 & 13.62 & 1.45 & - \\
CNT-N/S & 89.68 & 8.15 & 0.74 & 1.43 \\
\hline
\end{tabular}



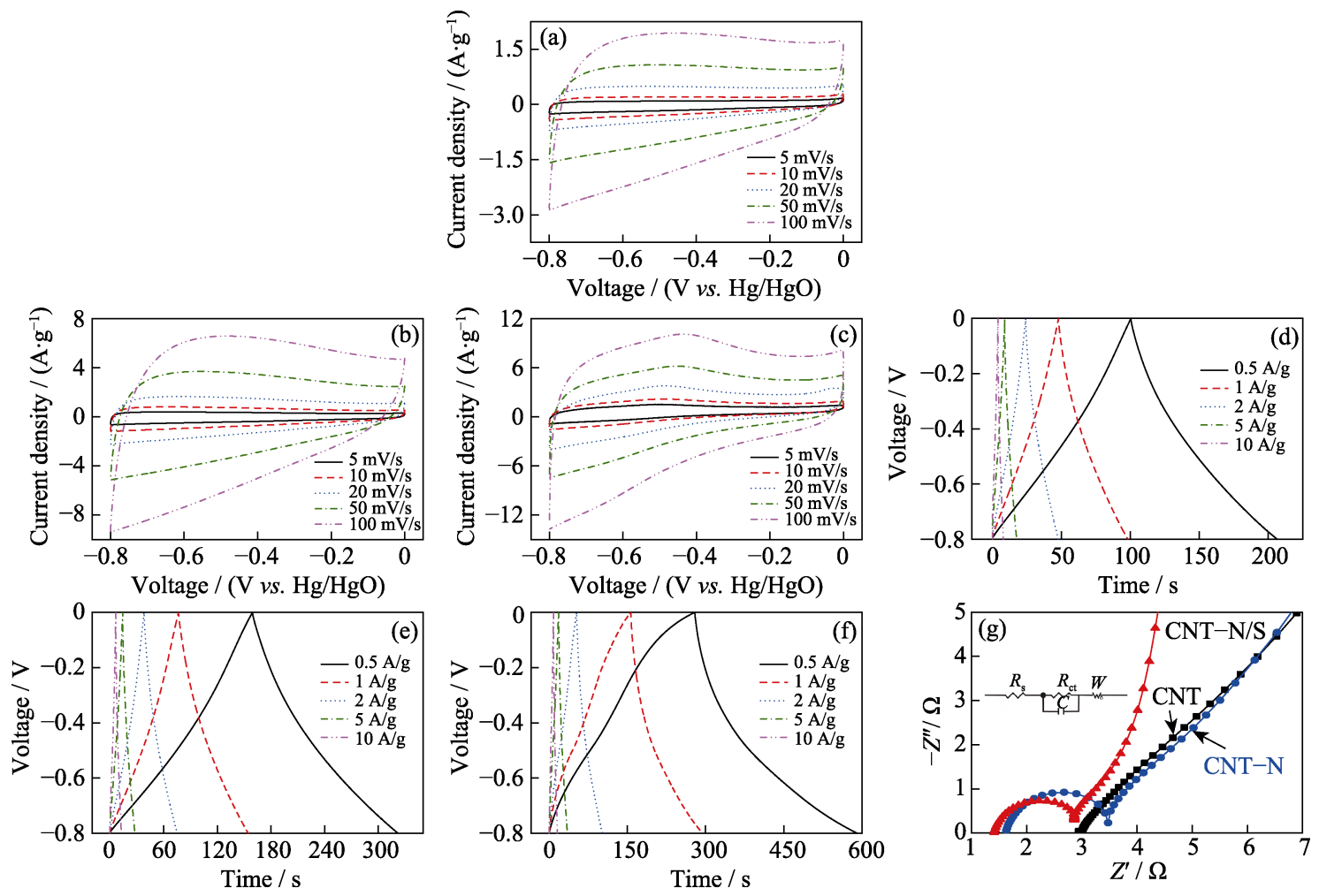

图 S4 (a, d)CNT、 $(b, e) C N T-N$ 和 $(c, f) C N T-N / S$ 电极 $(a \sim c)$ 在不同扫描速率下的循环伏安曲线和 $(d \sim f)$ 在不同电流密度下的 恒电流充放电曲线; CNT、CNT-N 和 CNT-N/S 电极的(g)Nyquist 曲线(内插图为等效电路图)

Fig. S4 (a-c) CV curves at different scan rates and (d-f) GCD curves at different current densities of (a, d) CNT, (b, e) CNT-N and (c, f) CNT-N/S; (g) Nyquist plots of CNT, CNT-N and CNT-N/S with insert showing the corresponding equivalent circuit 图中的插图是根据 $\mathrm{EIS}$ 拟合的等效电路图, 其中是 $R_{\mathrm{s}}$ 为溶液电阻、 $R_{\mathrm{ct}}$ 为界面电荷转移电阻, $W$ 为 Warburg 阻抗, $C$ 为双电层电容 ${ }^{[2]}$

(a)

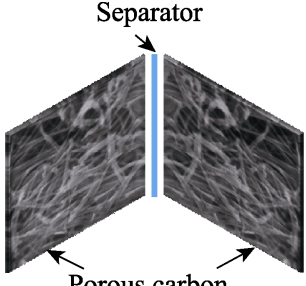

Porous carbon
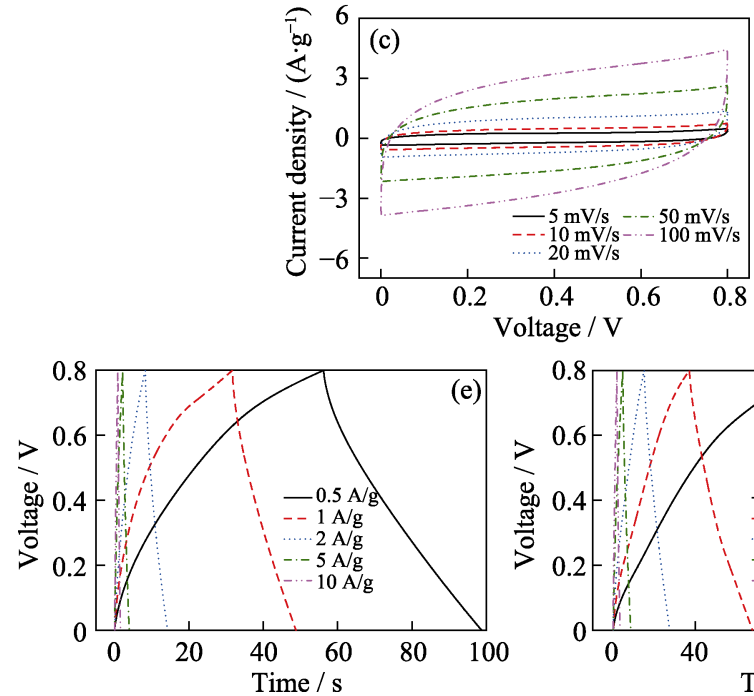
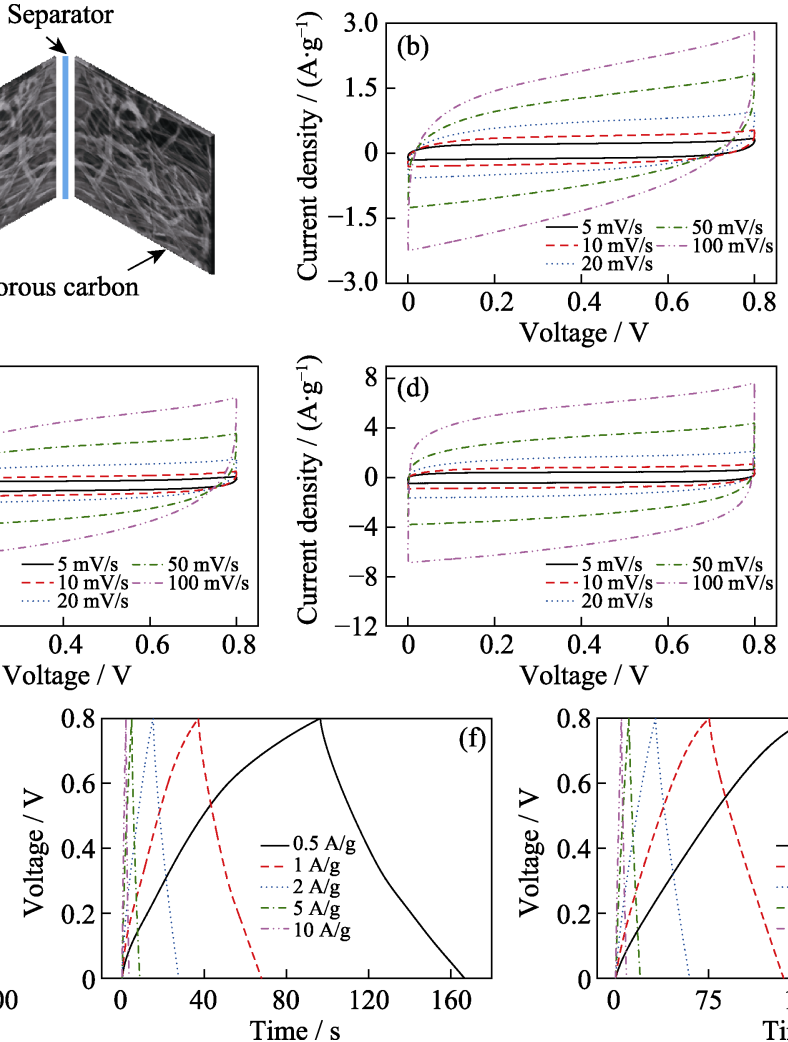

图 S5 (a)对称超级电容器的结构示意图; (b, e)CNT//CNT、 (c, f)CNT-N//CNT-N 和 $(d, g) C N T-N / S / / C N T-N / S$ (b d) 在不同 扫描速率下的循环伏安曲线和 $(\mathrm{e} \sim \mathrm{g})$ 在不同电流密度下的恒电流充放电曲线

Fig. S5 (a) Schematic structure of symmetric capacitor; (b-d) CV curves at different scan rates and (e-g) GCD curves at different current densities of (b, e) CNT//CNT, (c, f) CNT-N//CNT-N and (d, g) CNT-N/S//CNT-N/S 


\section{参考文献:}

[1] MIAO L, ZHU D Z, LIU M X, et al. Cooking carbon with protic salt: nitrogen and sulfur self-doped porous carbon nanosheets for supercapacitors. Chemical Engineering Journal, 2018, 347:
233-242.

[2] LI A J, CHUAN X Y, YANG Y, et al. Influence of activated condition on the structure of diatomite-templated carbons and their electrochemical properties as supercapacitors. Electrochemistry, 2017, 85(11): 708-714. 